\title{
Pretreatment Features to Influence Effectiveness of Intravesical Hyaluronic Acid Instillation in Refractory Interstitial Cystitis/ Painful Bladder Syndrome
}

\author{
Aram Kim, Bumjin Lim, Miho Song, Myung-Soo Choo \\ Department of Urology, Asan Medical Center, University of Ulsan College of Medicine, Seoul, Korea
}

Purpose: To determine the efficacy of intravesical hyaluronic acid (HA) instillation in treating patients with refractory interstitial cystitis/painful bladder syndrome (IC/PBS) and to identify any related factors that influence its therapeutic effect.

Methods: Thirty-three female IC/PBS patients who demonstrated poor or unsatisfactory responses to previous treatments between December 2010 and October 2012 were enrolled. Despite previous treatments, the enrolled patients had visual analogue scale (VAS) pain scores $\geq 4$ and total scores (symptom and bother scores) $\geq 13$ on the pelvic pain and urgency/frequency (PUF) questionnaire and $\geq 12$ on the O'Leary-Sant interstitial cystitis symptoms index (ICSI)/problems index (ICPI). All patients received once weekly intravesical instillations of 40-mg HA diluted in 50-mL saline for 4 weeks. The efficacy of the HA instillation was evaluated by comparing the mean changes in the scores of the VAS and questionnaires from baseline to 4 weeks after treatment. Improvement was defined as a $\geq 2$ decrease in the VAS. Moreover, we investigated the effects of the presence of Hunner's ulcer and previous treatment modalities on the therapeutic outcome of HA instillation.

Results: The mean age was $57.0 \pm 1.8$ years (range, $28-75$ years). The VAS score significantly decreased from baseline to 4 weeks after treatment $(-2.5, \mathrm{P}<0.001)$. The mean changes in the PUF, ICSI, and ICPI from baseline to 4 weeks after the treatment were $-3.8(\mathrm{P}<0.001),-2.3(\mathrm{P}<0.001)$, and $-2.7(\mathrm{P}<0.001)$, respectively. Twenty patients $(61 \%)$ showed improvements. Previous treatment modalities did not affect the efficacy of HA instillation and the presence of Hunner's ulcer was unrelated to outcomes. No complications were observed.

Conclusions: These results show that intravesical HA instillation is an effective and safe treatment for patients with refractory IC/PBS. Previous treatment modalities and presence of Hunner's ulcer do not affect the efficacy of HA instillation.

Keywords: Hyaluronic acid; Intravesical administration; Interstitial cystitis

\section{INTRODUCTION}

Interstitial cystitis/painful bladder syndrome (IC/PBS) is characterized by urinary frequency, urgency, and pelvic pain in the absence of any other identifiable pathology, such as infection or bladder cancer $[1,2]$. Therefore it is defined by the International Continence Society as "The complaint of suprapubic pain, related to bladder filling accompanied by other symptoms, such as increased day time and night time frequency, in the absence of proven urinary tract infection or other obvious pathology" of the lower urinary tract [3]. The prevalence of IC/PBS is approximate- ly 450 per 100,000 women [4]. Severe cases account for about $10 \%$ of the total and most studies show a female to male dominance of 5:1 [5]. The prevalence of IC/PBS in South Korean women (261/ 100,000 or 0.26\%) is lower than that of Europe and the United States and similar to that of Japan [6]. IC/PBS can result in sleep dysfunction, depression, anxiety and sexual problem, so it impairs quality of life [7].

Treatment modalities of IC/PBS are oral medicines, intravesical drug instillation, neuro-modulation, and surgical therapy such as hydrodistension and transurethral cauterization of Hunner's ulcer (TUC). Despite many treatment strategies, there are
Corresponding author: Myung-Soo Choo

Department of Urology, Asan Medical Center, University of Ulsan College of Medicine, 88 Olympic-ro 43-gil, Songpa-gu, Seoul 138-736, Korea

Tel: +82-2-3010-3734 / Fax: +82-2-477-8928 / E-mail: mschoo@amc.seoul.kr Submitted: July 4, 2014 / Accepted after revision: September 17, 2014
This is an Open Access article distributed under the terms of the Creative Commons Attribution Non-Commercial License (http://creativecommons.org/licenses/by-nc/3.0/) which permits unrestricted non-commercial use, distribution, and reproduction in any medium, provided the original work is properly cited. 
no standard treatment guidelines. Many drugs and procedures are empirically used, though only a few have been studied in randomized controlled trials [8]. Even though several treatments have been proposed for IC/PBS, IC/PBS cases often show recurrent and refractory characteristics. Thus, IC/PBS deserves to be challenged in the urological practice.

IC/PBS could be pathophysiologically related to disruption of the bladder mucosa surface layer and the consequent loss of glycosaminoglycans (GAGs), a class of mucopolysaccharides with hydrorepellent properties, thereby exposing the urothelium to toxic urinary agents [9]. Intravesical instillation of hyaluronic acid (HA) may regenerate the damaged GAG layer of the bladder in patients with IC/PBS [10]. Several studies reported that HA instillation is an effective treatment for IC/PBS [11-13] and the efficacy of HA instillation was reported $30 \%-70 \%$ improvement rates [12-14]. However there are not many studies that reported long-term efficacy of HA instillation. In the 5 years follow-up study, $50 \%$ of patients (24/48) showed complete bladder symptom remission without any additional therapy [15]. In our current study, we assessed the efficacy of HA instillation for refractory IC/PBS patients and determined the effects of previous treatments and Hunner's ulcer on clinical outcomes.

\section{MATERIALS AND METHODS}

We enrolled 33 female IC/PBS patients who were refractory to previous treatments with a visual analogue scale (VAS) pain score $\geq 4$ and total scores (symptom and bother score) $\geq 13$ on the pelvic pain and urgency/frequency (PUF) questionnaire and $\geq 12$ on the O'Leary-Sant interstitial cystitis symptoms index (ICSI)/problems index (ICPI). IC/PBS was diagnosed using East Asian Guidelines according to the characteristic symptoms of suprapubic pain, urinary frequency, and nocturia and cystoscopic findings [16]. We excluded patients who had bladder cancer, bladder stones, recurrent urinary tract infection, previous history of HA instillation, and bladder instillation of anticancer agents. We used our institution protocol of HA instillation: patients received intravesical instillations of HA once weekly for 4 weeks. The instillation was performed by $40-\mathrm{mg}$ HA diluted in $50-\mathrm{mL}$ saline for 30 minutes. Afterward, according to patient consultations, two more instillations were performed monthly in selected patients.

We evaluated the efficacy by determining the mean changes in the VAS pain score from baseline to 4 weeks after the HA treatment as a primary end point [17]. The secondary end points were the changes in the PUF [18] and ICSI/ICPI as reported on the IC/PBS questionnaires. Improvement was considered to be a $\geq 2$ decrease in VAS according to our protocol. We evaluated the correlation between the previous treatment modalities, presence of Hunner's ulcer, and the efficacy of HA instillation.

Statistical analysis of questionnaire data was performed using analysis of variance to assess dependent samples. The statistical significance of the changes from baseline was assessed using the paired t-test. All the statistical analysis was performed using the SPSS ver. 15.0 (SPSS Inc., Chicago, IL, USA) and $\mathrm{P}<0.05$ was considered statistically significant.

\section{RESULTS}

The mean age was $57.0 \pm 1.8$ years (range, $28-75$ years) and the demographic characteristics of the patients are shown in Table 1. Regarding previous treatments, 18 patients (54.5\%) took pentosan polysulfate (PPS), 10 patients (30.3\%) underwent hydrodistension, and 5 patients (15.1\%) underwent TUC. At baseline, the mean VAS score was 5.1, the PUF-symptom score was 12.8 , the PUF-bother score was 7.2 , the ICSI score was 12.5 , and the ICPI score was 11.4. Ten patients (30.3\%) had Hunner's ulcer.

After 4 weeks, the change in the VAS pain score was -2.5 $(\mathrm{P}<0.001)$. The PUF-total score $(-3.8, \mathrm{P}<0.001)$, ICSI $(-2.3, \mathrm{P}<$ $0.001)$, and ICPI $(-2.7, \mathrm{P}<0.001)$ also showed significant improvements (Fig. 1). Twenty patients (61.0\%) showed improvements after 4 weeks of HA instillation (VAS from 7.0 to 4.0), eleven patients (33.3\%) who had one or two additional HA instillations also showed improvements (VAS from 4.6 to 2.0). Spe-

Table 1. Patient characteristics $(\mathrm{n}=33)$

\begin{tabular}{lc}
\hline Characteristic & Value \\
\hline Mean age (yr) & $57.0 \pm 1.8$ \\
Previous treatment & \\
Pentosan polysulfate & 18 \\
Hydrodistension & 10 \\
Transurethral cauterization & 5 \\
Baseline & \\
VAS score & $5.1 \pm 0.5$ \\
PUF-symptom score & $12.8 \pm 0.8$ \\
PUF-bother score & $7.2 \pm 0.3$ \\
ICSI & $12.5 \pm 0.7$ \\
ICPI & $11.4 \pm 0.5$ \\
\hline
\end{tabular}

Values are presented as mean \pm standard deviation.

PUF, pelvic pain and urgency/frequency questionnaire; ICSI, O'LearySant interstitial cystitis symptoms index; ICPI, O'Leary-Sant interstitial cystitis problem index. 
Table 2. Mean changes in variables from baseline to after 4 weeks of hyaluronic acid treatment according to previous treatment

\begin{tabular}{lcccc}
\hline Variable & Pentosan polysulfate & Hydrodistension & TUC & P-value \\
\hline VAS pain scores & $-3.1 \pm 0.2$ & $-3.1 \pm 0.2$ & $-2.3 \pm 0.1$ & 0.982 \\
Frequency/day & $-3.8 \pm 0.4$ & $-3.0 \pm 0.2$ & $-3.8 \pm 0.4$ & 0.973 \\
PUF patient symptom scale & & & & \\
Symptom score & $-2.2 \pm 0.3$ & $-2.0 \pm 0.2$ & $-2.2 \pm 0.3$ & 0.903 \\
Bother score & $-1.6 \pm 0.1$ & $-1.6 \pm 0.2$ & $-1.3 \pm 0.3$ & 0.446 \\
Total score & $-3.9 \pm 0.4$ & $-3.6 \pm 0.4$ & $-3.9 \pm 0.4$ & 0.709 \\
O'Leary-Sant IC index & & & & 0.245 \\
ICSI & $-2.6 \pm 0.3$ & $-2.5 \pm 0.3$ & $-2.6 \pm 0.4$ & 0.824 \\
ICPI & $-2.5 \pm 0.3$ & $-2.1 \pm 0.3$ & $-2.5 \pm 0.4$ & \\
\hline
\end{tabular}

Values are presented as mean \pm standard deviation.

TUC, transurethral cauterization of Hunner's ulcer; VAS, visual analogue scale; PUF, pelvic pain and urgency/frequency; IC, interstitial cystitis; ICSI, O'Leary-Sant interstitial cystitis symptoms index; ICPI, O'Leary-Sant interstitial cystitis problem index.

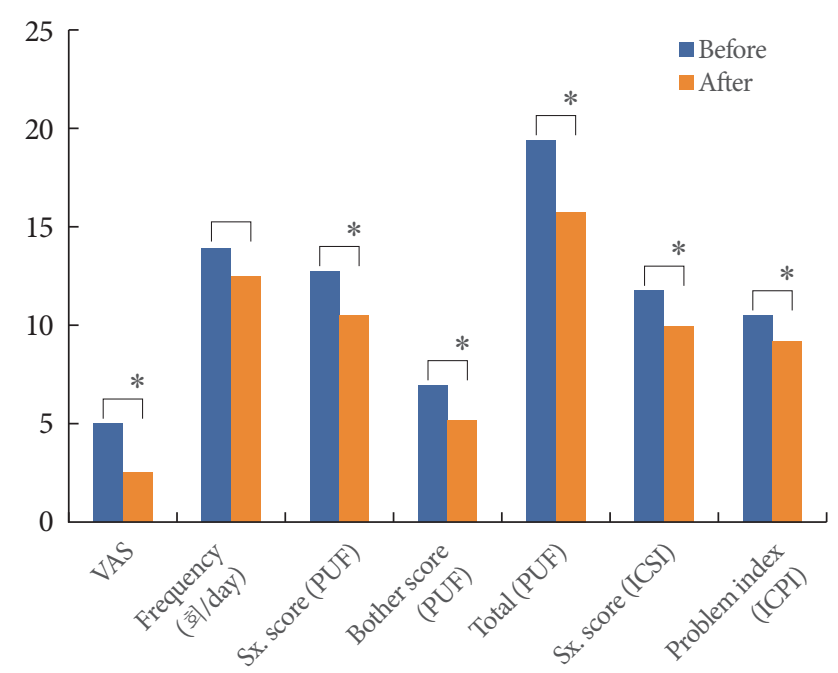

Fig. 1. Mean changes in variables from baseline to 4 weeks after hyaluronic acid treatment. VAS, visual analogue scale; PUF, pelvic pain and urgency/frequency; ICSI, interstitial cystitis symptoms index; ICPI, interstitial cystitis problems index. ${ }^{*} \mathrm{P}<0.05$.

cifically, HA instillation significantly improved voiding symptoms according to questions 1 and 3 in the ICPI $(\mathrm{P}<0.001$ and $\mathrm{P}=0.026$, respectively) and bladder pain and nocturia according to questions 2 and 4 in the ICPI $(\mathrm{P}=0.026$ and $\mathrm{P}=0.008$, respectively). However, outcomes of sexual function did not improve significantly according to question 4 in the PUF $(\mathrm{P}=0.771)$. Improvements were maintained for 11.1 weeks (range, 2-40 weeks) in these patients after the last HA instillation.

According to the previous treatment modalities, there were no differences in efficacy in terms of VAS pain, frequency, PUFtotal score, ICSI, or ICPI (Table 2). There were no significant differences in the mean changes in the VAS pain score, fre-
Table 3. Mean change in variables from baseline to 4 weeks after hyaluronic acid treatment in IC/PBS patients with or without Hunner's ulcer

\begin{tabular}{lccc}
\hline Variable & $\begin{array}{c}\text { With } \\
\text { Hunner's ulcer }\end{array}$ & $\begin{array}{c}\text { Without } \\
\text { Hunner's ulcer }\end{array}$ & P-value \\
\hline VAS pain scores & $-2.4 \pm 0.2$ & $-2.5 \pm 0.2$ & 0.148 \\
Frequency/day & $-2.0 \pm 0.1$ & $-2.3 \pm 0.2$ & 0.154 \\
PUF patient symptom scale & & & \\
$\quad$ Symptom score & $-2.6 \pm 0.3$ & $-2.7 \pm 0.3$ & 0.331 \\
$\quad$ Bother score & $-1.5 \pm 0.1$ & $-1.8 \pm 0.1$ & 0.145 \\
Total score & $-4.0 \pm 0.4$ & $-4.5 \pm 0.5$ & 0.327 \\
O'Leary-Sant index score & & & \\
$\quad \begin{array}{l}\text { Symptom index } \\
\text { Problem index }\end{array}$ & $-2.8 \pm 0.2$ & $-2.9 \pm 0.2$ & 0.186 \\
\hline
\end{tabular}

Values are presented as mean \pm standard deviation.

IC/PBS, interstitial cystitis/painful bladder syndrome; VAS, visual analogue scale; PUF, pelvic pain and urgency/frequency.

quency, PUF-total score, ICSI, or ICPI between patients with or without Hunner's ulcer (Table 3). No complications developed after HA instillation.

\section{DISCUSSION}

We here focused on refractory IC/PBS and proposed an additional HA instillation for the refractory IC/PBS in this study. We show that HA instillation improved VAS pain and IC/PBS questionnaire scores in refractory IC/PBS patients.

Several IC/PBS treatment modalities have been reported. The most commonly used three kinds of treatment were PPS, hydrodistension and TUC, and these treatment modalities showed some effects. However the efficacies of these treatment modali- 
ties were controversial because of the recurrence and the complications. The treatment response of PPS was about half and many of these patients needed additional treatments [19]. In one study, $72.4 \%$ of patients needed renewed instillation or maintenance therapy [20]. Hydrodistension has been used for a long time. The improvement by hydrodistension disappeared within 3 to 6 months [21]. Moreover, hydrodistension can have diverse adverse effects, such as hematuria, bladder pain, and bladder rupture [22]. TUC is an option for IC/PBS patients with Hunner's ulcer. But, it has a problem of high rate of recurrence. One group reported $45.8 \%$ of patients required repeated fulguration after a median follow-up of 44.8 months, indicating that this procedure does not cure the underlying disease and only improves the symptoms [23].

As mentioned above, in our IC/PBS patients the symptoms have recurred after these treatments [24]. For this reason, we need to focus on additional treatment modality for refractory IC/PBS which was defined as persistent, unacceptable symptoms despite PPS, hydrodistension, and TUC treatment [8].

HA is considered a good candidate for GAG substitution and used in the treatment of patients with recurrent cystitis, hemorrhagic cystitis and IC in urology field [25]. There were several reports of HA instillation for IC/PBS, one study reported a satisfactory response of IC/PBS patients to intravesical HA instillation for 4 weeks [26]. Another study showed acute remission and long-term HA efficacy in the treatment of IC/PBS and HA instillation was considered to improve functional bladder capacity, symptom scores, pain, and quality of life. The average initial VAS score for all patients was 8.15 , which decreased to $2.71 \mathrm{im}$ mediately after HA therapy and stayed stable at 6 months after instillation therapy with an average score of 2.7. A further reduction to 2.14 was seen 5 years later. A VAS score reduction of $>2$ was observed in $85.4 \%$ patients (41/48) [15]. However there is no study of HA instillation in patients with refractory IC/PBS. We focused treatment of HA in refractory IC/PBS patients.

In our present study, the VAS score significantly decreased from baseline to 4 weeks after treatment $(-2.5)$ and the PUF and ICSI/ICPI questionnaires showed significant improvements after 4 weeks of HA instillation. The rate of improvement was $61 \%$, which was slightly lower than that of the previous reported study [15], probably because the patients in our study were refractory to conventional therapy. Eleven patients we analyzed had one or two additional HA instillations after the initial four HA instillations and showed continuous improvement (VAS from 4.6 to 2.0). Our instillation protocol was once weekly in- travesical instillations of HA for 4 weeks and two more instillations were performed monthly according to patient consultation. This protocol was similar that of other groups. In the literature, several different protocols have been described for instillation. Many uncontrolled studies used 40-mg HA dissolved in 40 $\mathrm{mL}$ of normal saline solution weekly for 4 to 6 weeks and then monthly $[8,13,26]$. One Danish uncontrolled pilot study enrolled patients with IC/PBS who received weekly bladder instillations of HA for 1 month and monthly instillations for an additional 2 months [27].

Furthermore, there were no differences in the efficacy of HA instillation according to previous treatment modalities and there was no effect of Hunner's ulcer on HA instillation efficacy. Because Hunner's ulcer already removed by TUC, there was no difference in HA instillation efficacy between the presence and absence of Hunner's ulcer.

No adverse event for HA instillation were reported in our present study. There has been no report on the serious adverse events from HA instillation, but one study has mentioned mild bladder irritation [25]. The limitations of our current investigation include the short study duration and follow-up period, small population, and retrospective design.

In conclusion, instillation of HA is a feasible treatment option for refractory IC/PBS patients who have failed to respond to previous treatment. The previous treatment modalities and presence of Hunner's ulcer do not influence the outcomes of HA instillation.

\section{CONFLICT OF INTEREST}

No potential conflict of interest relevant to this article was reported.

\section{REFERENCES}

1. Dell JR. Interstitial cystitis/painful bladder syndrome: appropriate diagnosis and management. J Womens Health (Larchmt) 2007;16: 1181-7.

2. Gulpınar O, Haliloglu AH, Gokce MI, Arıkan N. Instillation of hyaluronic acid via electromotive drug administration can improve the efficacy of treatment in patients with interstitial cystitis/painful bladder syndrome: a randomized prospective study. Korean J Urol 2014;55:354-9.

3. Abrams P, Cardozo L, Fall M, Griffiths D, Rosier P, Ulmsten U, et al. The standardisation of terminology in lower urinary tract function: 
report from the standardisation sub-committee of the International Continence Society. Urology 2003;61:37-49.

4. Leppilahti M, Tammela TL, Huhtala H, Auvinen A. Prevalence of symptoms related to interstitial cystitis in women: a population based study in Finland. J Urol 2002;168:139-43.

5. Kusek JW, Nyberg LM. The epidemiology of interstitial cystitis: is it time to expand our definition? Urology 2001;57(6 Suppl 1):95-9.

6. Choe JH, Son H, Song YS, Kim JC, Lee JZ, Lee KS. Prevalence of painful bladder syndrome/interstitial cystitis-like symptoms in women: a population-based study in Korea. World J Urol 2011;29: 103-8.

7. Nickel JC, Tripp DA, Pontari M, Moldwin R, Mayer R, Carr LK, et al. Psychosocial phenotyping in women with interstitial cystitis/ painful bladder syndrome: a case control study. J Urol 2010;183: 167-72.

8. Ham BK, Kim JH, Oh MM, Lee JG, Bae JH. Effects of combination treatment of intravesical resiniferatoxin instillation and hydrodistention in patients with refractory painful bladder syndrome/interstitial cystitis: a pilot study. Int Neurourol J 2012;16:41-6.

9. Parsons CL, Hurst RE. Decreased urinary uronic acid levels in individuals with interstitial cystitis. J Urol 1990;143:690-3.

10. Lokeshwar VB, Selzer MG, Unwala DJ, Estrella V, Gomez MF, Golshani $\mathrm{R}$, et al. Uronate peaks and urinary hyaluronic acid levels correlate with interstitial cystitis severity. J Urol 2006;176:1001-7.

11. Porru D, Leva F, Parmigiani A, Barletta D, Choussos D, Gardella B, et al. Impact of intravesical hyaluronic acid and chondroitin sulfate on bladder pain syndrome/interstitial cystitis. Int Urogynecol J 2012;23:1193-9.

12. Morales A, Emerson L, Nickel JC. Intravesical hyaluronic acid in the treatment of refractory interstitial cystitis. Urology 1997 May;49(5A Suppl):111-3.

13. Porru D, Campus G, Tudino D, Valdes E, Vespa A, Scarpa RM, et al. Results of treatment of refractory interstitial cystitis with intravesical hyaluronic acid. Urol Int 1997;59:26-9.

14. Offiah I, McMahon SB, O’Reilly BA. Interstitial cystitis/bladder pain syndrome: diagnosis and management. Int Urogynecol J 2013;24: 1243-56.

15. Engelhardt PF, Morakis N, Daha LK, Esterbauer B, Riedl CR. Longterm results of intravesical hyaluronan therapy in bladder pain syndrome/interstitial cystitis. Int Urogynecol J 2011;22:401-5.
16. Homma Y, Ueda T, Tomoe H, Lin AT, Kuo HC, Lee MH, et al. Clinical guidelines for interstitial cystitis and hypersensitive bladder syndrome. Int J Urol 2009;16:597-615.

17. Butrick CW. Interstitial cystitis and chronic pelvic pain: new insights in neuropathology, diagnosis, and treatment. Clin Obstet Gynecol 2003;46:811-23.

18. Son HC, Jung YJ, Chang JS, Kim SH, Hong SK, Oh SJ, et al. Translation and linguistic validation of the korean version of the pelvic pain and urgency/frequency patient symptom scale. Int Neurourol J 2010;14:112-21.

19. Nickel JC, Barkin J, Forrest J, Mosbaugh PG, Hernandez-Graulau J, Kaufman D, et al. Randomized, double-blind, dose-ranging study of pentosan polysulfate sodium for interstitial cystitis. Urology 2005; 65:654-8.

20. Daha LK, Lazar D, Simak R, Pfluger H. The effects of intravesical pentosanpolysulfate treatment on the symptoms of patients with bladder pain syndrome/interstitial cystitis: preliminary results. Int Urogynecol J Pelvic Floor Dysfunct 2008;19:987-90.

21. Shao Y, Shen ZJ, Rui WB, Zhou WL. Intravesical instillation of hyaluronic acid prolonged the effect of bladder hydrodistention in patients with severe interstitial cystitis. Urology 2010;75:547-50.

22. Hanno PM, Burks DA, Clemens JQ, Dmochowski RR, Erickson D, Fitzgerald MP, et al. AUA guideline for the diagnosis and treatment of interstitial cystitis/bladder pain syndrome. J Urol 2011;185:2162-70.

23. Hillelsohn JH, Rais-Bahrami S, Friedlander JI, Okhunov Z, Kashan M, Rosen L, et al. Fulguration for Hunner ulcers: long-term clinical outcomes. J Urol 2012;188:2238-41.

24. Ryu J, Pak S, Song M, Chun JY, Hong S, Choo MS. Elimination of Hunner's ulcers by fulguration in patients with interstitial cystitis: is it effective and long lasting? Korean J Urol 2013;54:767-71.

25. Iavazzo C, Athanasiou S, Pitsouni E, Falagas ME. Hyaluronic acid: an effective alternative treatment of interstitial cystitis, recurrent urinary tract infections, and hemorrhagic cystitis? Eur Urol 2007; 51:1534-40.

26. Morales A, Emerson L, Nickel JC, Lundie M. Intravesical hyaluronic acid in the treatment of refractory interstitial cystitis. J Urol 1996; 156:45-8.

27. Kallestrup EB, Jorgensen SS, Nordling J, Hald T. Treatment of interstitial cystitis with cystistat: a hyaluronic acid product. Scand J Urol Nephrol 2005;39:143-7. 